\title{
2
}

\section{INCLUSIÓN DE PERSONAS CON DISCAPACIDAD EN EL MEDIO LABORAL}

Paulina Guerra, Carlos Ramos, Andrés Subia, Victor Carvajal, Santiago Borja 


\title{
INCLUSIÓN DE PERSONAS CON DISCAPACIDAD EN EL MEDIO LABORAL
}

\author{
Paulina Guerra, Carlos Ramos, Andrés Subía, Víctor Carvajal y Santiago Borja \\ Escuela de Psicología. Universidad Tecnológica Indoamérica. \\ paulinaguerra@uti.edu.ec
}

\begin{abstract}
Resumen
En el presente artículo reportamos una investigación que analizó la inclusión de personas con discapacidad en el medio laboral. El primer paso seguido en el proceso metodológico fue la construcción de un instrumento para cuantificar el nivel de conocimiento del proceso inclusivo que los trabajadores poseen sobre las personas con discapacidad. La muestra del estudio estuvo conformada por 150 participantes (95 mujeres y 55 hombres). El diseño de investigación se basó en un método cuantitativo con un alcance descriptivo. La estadística se basó en un proceso de medidas de tendencia central y dispersión. Como resultados encontramos que la mayoría de participantes conocen sobre las discapacidades y su proceso de inclusión laboral. Como investigación futura nos planteamos analizar el rendimiento de personas con discapacidad que han sido incluidas en algún determinado contexto laboral.
\end{abstract}

Palabras claves: discapacidad, inclusión laboral, procesos de selección.

\begin{abstract}
In this article we report an investigation that analyzed the inclusion of persons with disabilities in the workplace. The first step in the methodological process followed was the construction of an instrument for measuring the level of knowledge of the inclusive process that workers have on people with disabilities. The study sample consisted of 150 participants (95 women and 55 men). The research design was based on a quantitative method with a descriptive scope. The statistics are based on a process of measures of central tendency and dispersion. As a result we found that most participants know about disabilities and labor inclusion process. As future research was to analyze the performance of people with disabilities who have been included in any particular work context.
\end{abstract}

Keywords: disability, labor inclusion, selection processes. 


\section{INTRODUCCIÓN}

La Organización Mundial de la Salud (OMS), define al concepto de discapacidad como un término general que abarca las deficiencias, las limitaciones de la actividad y las restricciones de la participación. Las deficiencias son problemas que afectan a una estructura o función corporal; las limitaciones de la actividad son dificultades para ejecutar acciones o tareas, y las restricciones de la participación son problemas para participar en situaciones vitales. [1].

Después de la aprobación del modelo de inclusión a la sociedad de las personas con discapacidad en el año 2006, se define a las discapacidades como un concepto que evoluciona y que resulta de la interacción entre las personas con deficiencias y las barreras debidas a la actitud y al entorno que evitan su participación plena y efectiva en la sociedad, en igualdad de condiciones con las demás. [2]

De acuerdo a los datos obtenidos en el Registro Nacional de Discapacidades de Ecuador CONADIS [3], podemos identificar estadísticas agrupadas por tipo de discapacidad de la siguiente manera, discapacidad física, discapacidad psicológica, discapacidad Intelectual, discapacidad Sensorial (Visual, Auditiva y de Lenguaje).

La discapacidad física se refiere a las deficiencias corporales y/o viscerales que ocasionan dificultad significativa o imposibilidad para caminar, correr, manipular objetos con las manos, subir o bajar gradas, levantarse, sentarse, mantener el equilibrio, controlar esfínteres, entre otras [3].

La discapacidad psicológica se refiere a las deficiencias y/o trastornos del comportamiento, del razonamiento, de los estados de ánimo, crónicos y permanentes que afectan su capacidad de juicio crítico sobre sí mismo y sobre los demás [3].

La discapacidad intelectual se refiere a las deficiencias cognitivas que dificultan la comprensión de ideas complejas, así como la capacidad de razonar, de abstracción y de anticipar los peligros. La característica principal es la dificultad para aprender $\mathrm{y}$ poder ejecutar algunas actividades de la vida cotidiana [3].

La discapacidad visual se refiere a personas que presentan ceguera y baja visión. En ambas situaciones estaríamos hablando de personas con un alto grado de pérdida de visión, que causa dificultad en la movilidad y la orientación [3].

La discapacidad auditiva se refiere a personas que presentan sordera debido a que no han desarrollado el sentido del oído o han perdido la capacidad de escuchar, situación que dificulta la comunicación con su entorno. Muchos de estos casos se ven acompañados por la ausencia de lenguaje, circunstancia que requiere del uso de audífonos y la expresión mediante la lengua de señas [3].

La discapacidad del lenguaje se refiere a personas que presentan deficiencia para la expresión verbal que dificulta la comunicación y la interrelación; puede producirse de manera vinculada a la sordera, o ser una secuela de otro tipo de lesiones [3].

Las personas con discapacidad representan aproximadamente mil millones de personas, lo cual sería un $15 \%$ de la población mundial, donde alrededor del $80 \%$ están en edad de trabajar. Sin embargo, su derecho a un trabajo digno, es con frecuencia denegado. Las personas con discapacidad, en particular las mujeres con discapacidad, se enfrentan a enormes barreras actitudinales, físicas y de la información que dificultan el disfrute a la igualdad de oportunidades en el mundo del trabajo. En comparación con las personas sin discapacidad, las personas con discapacidad experimentan mayores tasas de desempleo e inactividad económica y están en mayor riesgo de una protección social insuficiente la cual es clave para reducir la pobreza extrema.[4]

Las personas con discapacidad se enfrentan a una de las tasas de desempleo más altas y una mayor probabilidad de ser económicamente inactivas que las personas sin discapacidad. Si están trabajando, tienen más probabilidades de tener empleos mal remunerados, con pocas perspectivas profesionales y malas condiciones de trabajo. Es así que, en el mundo existe un vínculo innegable entre la discapacidad, la pobreza y la exclusión. La falta de igualdad de oportunidades de empleo para las personas con discapacidad constituye una de las causas subyacentes de la pobreza y la exclusión de dichas personas.[5]

De acuerdo a un estudio realizado en Chile, los cargos que ocupan las personas con discapacidad en las empresas son de nivel jerárquico bajo. Casi la totalidad de la muestra (96\%) se desempeña como empleado u obrero, mientras que sólo un $2,4 \%$ lo hace como jefe o mando medio. El 1,6\% se desempeña en la alta dirección (gerencia o superior), presentando discapacidad física casi la totalidad de ellos. En coherencia con lo señalado, las áreas de trabajo que predominan son "operario" (31\%) y "personal de apoyo" (29\%). En una proporción menor aparecen "trabajos administrativos" y "otras áreas" (producción, profesionales). Sólo un 5,6\%labora en el área comercial y ventas [6]

Según la Organización Internacional del Trabajo (OIT) el $85 \%$ de las personas con discapacidad en edad de trabajar dentro de América Latina y el Caribe, no tienen un empleo. El Ecuador no es la excepción, más aún cuando las personas con múltiple discapacidad y la población con discapacidad visual se enfrentan al reto de estudiar 
para luego no conseguir un trabajo. Hace falta la colaboración de las empresas estatales y privadas para adaptar y adecuar un puesto de trabajo con la infraestructura física necesaria, así como para implementar ayudas técnicas (iluminación, máquinas de escritura en braille, manejo de Jaws, etc.[7]

Dentro del contexto descrito anteriormente surge la inquietud de analizar si existe algún tipo de diferenciación en la inclusión o selección de las personas con discapacidad en la población ecuatoriana. Dentro de este esquema, el objetivo principal de este trabajo de investigación es generar información necesaria que permita orientar a la sociedad sobre el funcionamiento del proceso de selección a personas con discapacidad en una muestra de personas entre estudiantes y profesionales residentes en el Distrito Metropolitano de Quito, y de esta manera correlacionar las variables cualitativas como género, edad y profesión con la encuesta aplicada para observar factores diferenciadores en los procesos de selección e inclusión de personas con discapacidad.

\section{MÉTODO}

\section{Participantes}

La muestra estuvo conformada por 150 personas entre ellas estudiantes universitarios y profesionales de la ciudad de Quito (95 mujeres y 55 hombres), Ecuador. Sus edades comprendieron entre los 15 y 60 años. Para la participación se establecieron tres criterios, edad, sexo y profesión. En la figura 1 presentamos la distribución porcentual del género de los participantes.

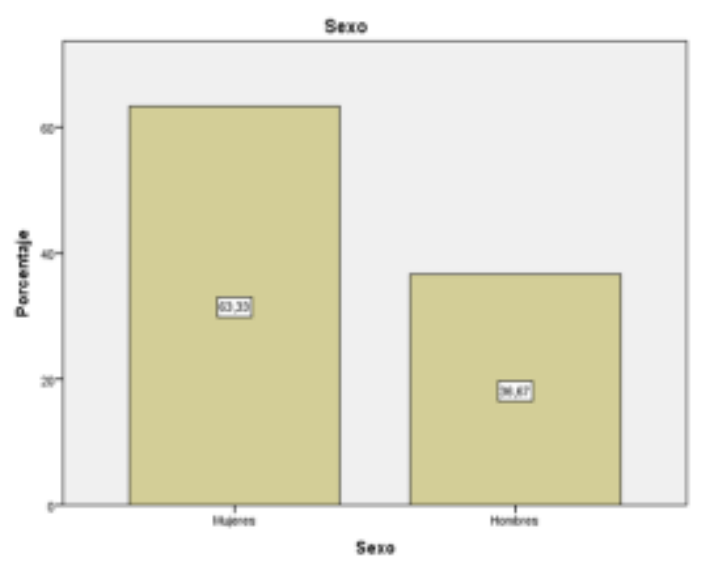

Figura 1.- El cuadro estadístico presentado nos muestra que el $63,3 \%$ de la población encuestada está representada por las mujeres, mientras que un $36,7 \%$ está representada por los hombres.

En cuanto a la edad de los participantes, 41 fueron de 15 a 20 años de edad, 56 de 21 a 30 años de edad, 21 de 31 a 40 años de edad, 26 de 41 a 50 años de edad, 6 de 51 a 60 años de edad. En la figura 2 se puede observar la distribución porcentual de la edad de los participantes.

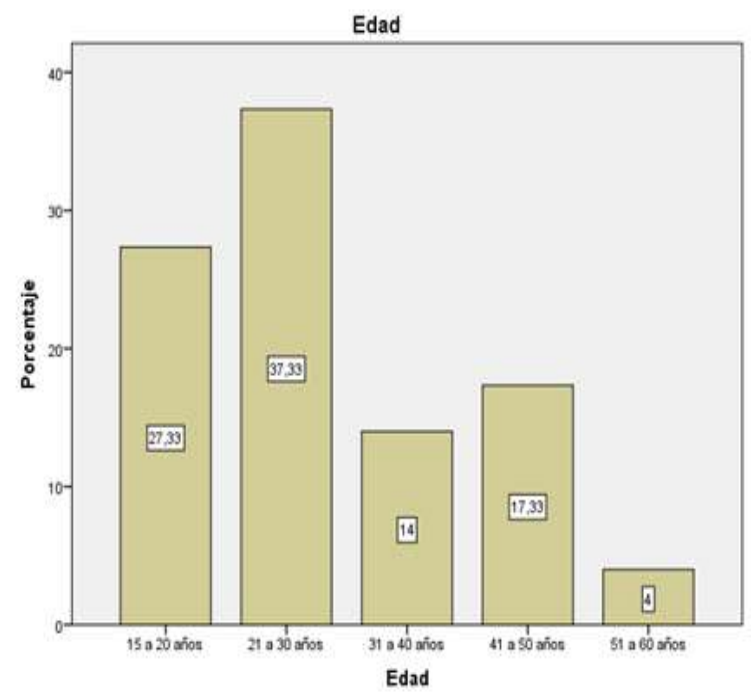

Figura 2.- El cuadro analítico nos presenta que el porcentaje más alto de edades de las personas encuestadas es de un $37,33 \%$ representada por las edades de entre 21 a 30 años, seguido por el $27,33 \%$ representada por edades de 15 a 20 años, mientras que los porcentajes bajos están en edades de 41 a 50 años con un $17,33 \%, 31$ a 40 años con un $14 \%$ y finalmente las edades de entre 51 a 60 años con un $4 \%$.

En referencia a la actividad de los participantes, 50 fueron estudiantes (33\%), 30 educadores (20\%), 20 gerentes (14\%), 16 comerciantes $(10 \%$, ingenieros $11(7 \%)$, médicos 15 (10\% y servidores públicos $8(5 \%)$.

\section{Diseño de Investigación}

Se trata de un método cuantitativo, no experimental, alcance descriptivo y temporalidad transversal.

\section{Instrumentos y Técnicas de Recolección de Datos}

Para la medición de las variables se construyó un cuestionario de 10 ítems que evaluó varios aspectos del proceso laboral de personas con 
discapacidad. En el apartado de resultados se presenta el contenido de cada ítem.

\section{Análisis de Datos}

Se utilizó estadística descriptiva de medidas de tendencia central y dispersión.

\section{RESULTADOS}

En primer lugar se presentarán los resultados descriptivos de cada una de las preguntas valoradas, posteriormente se expondrán los resultados del cruce de las variables de interés.

Los resultados descriptivos de la aplicación del instrumento fueron, en la pregunta uno que consultó ¿Conoce usted sobre las discapacidades? se encontró que el $94,7 \%$ de las personas encuestadas respondieron que sí conocen sobre las discapacidades, mientras que un 5,3\% respondieron no conocer sobre las discapacidades.

En la pregunta dos: ¿Conoce usted sobre la inclusión de las personas con discapacidad en el ámbito laboral? se encontró que el $84,7 \%$ de las personas encuestadas si conocen sobre la inclusión laboral de las personas con discapacidad, mientras que el $15,3 \%$ respondieron que no conocen sobre el tema.

En la pregunta tres: ¿Cree usted que una discapacidad es un impedimento para trabajar dentro de una organización? se observó que el 95,3\% de las personas encuestadas respondieron que la discapacidad no es un impedimento para trabajar, mientras que el $4,7 \%$ respondieron que sí.

En la pregunta cuatro: ¿Cree usted que las personas con discapacidad se limitan a ciertas áreas de trabajo? se encontró que el $18,7 \%$ de las personas encuestadas respondieron que las personas con discapacidad no se limitan a ciertas áreas de trabajo, mientras que un $81,3 \%$ respondieron que sí se limitan.

En la pregunta número cinco: ¿Cree usted que las personas con discapacidad deben tener un proceso de selección para inclusión laboral diferente a las establecidas para las demás personas? observamos que el $34,7 \%$ de las personas encuestadas dicen que las personas con discapacidad no deben tener un proceso de selección diferente a las establecidas, mientras que el $65,3 \%$ respondieron que sí deben tener un proceso diferente.

En la pregunta seis: ¿Conoce usted sobre los procesos de selección a personas con discapacidad?
Observamos que un $74,7 \%$ de las personas encuestadas dicen que no conocen sobre los procesos de selección a personas con discapacidad, mientras que un 25,3\% respondieron que sí conocen.

En la pregunta número siete. ¿Cree usted que las personas con discapacidad son susceptibles a un rechazo de parte de las organizaciones?

Podemos observar que el $11,3 \%$ de las personas encuestadas dicen que no las personas con discapacidad no son susceptibles al rechazo, mientras que un 88,7 respondieron que sí son susceptibles al rechazo.

En la pregunta número ocho: ¿Cree usted que una persona con discapacidad aportaría de manera efectiva en el crecimiento de una organización?

Podemos observar que el $9,3 \%$ de las personas encuestadas dicen que las personas con discapacidad no aportarían de manera efectiva a una organización, mientras que un $90,7 \%$ respondieron que sí aportarían de manera efectiva a la empresa.

En la pregunta número nueve: ¿Cómo cree usted que es el desempeño laboral de las personas con discapacidad?

Observamos que el $58,7 \%$ de la población encuestada respondió que el desempeño laboral de las personas con discapacidad es mayor, mientras que el $38 \%$ de la población respondió que es medio y el 3,3\% respondió que su desempeño es bajo.

En la pregunta número diez: ¿Cuál considera usted que sería la discapacidad con más problemática laboral? Podemos observar que el $68,7 \%$ de la población encuestada dijo que la discapacidad con más problemática laboral es la discapacidad intelectual, el $21,3 \%$ dijo que es la discapacidad física y el $10 \%$ la discapacidad sensorial.

Una vez descritos los resultados de cada una de las preguntas, se pasará a exponer los análisis de varias relaciones que se realizaron. En la pregunta número 1 se hizo una comparación en las respuestas que dieron las personas de sexo femenino con las personas de sexo masculino, en donde observamos que un porcentaje alto sobre el conocimiento de las discapacidades, está representada por las personas de sexo masculino, con un $98,18 \%$, mientras que las personas de sexo femenino menciona conocer sobre las discapacidades en un $92,62 \%$. Este resultado se 10 puede observar de forma gráfica en la figura 1 


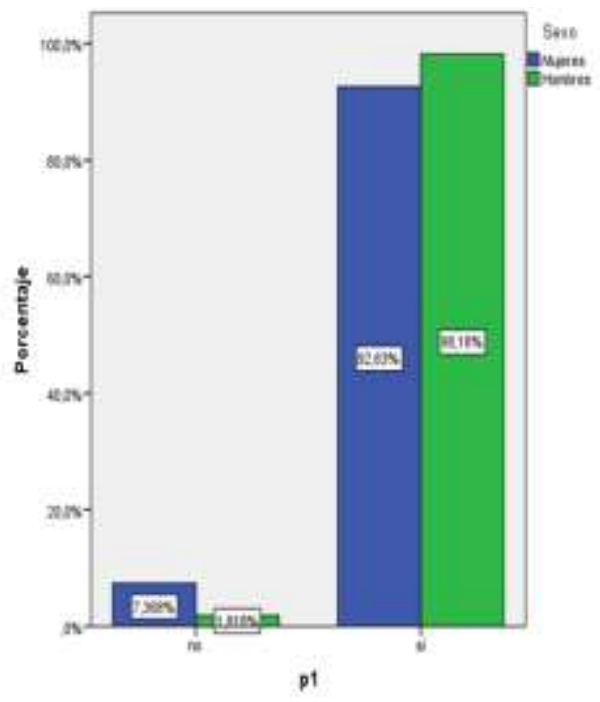

Figura 1.- En el cuadro presentado podemos observar que la respuesta SI está dada en un 92,63\% por los hombres y en un $98,18 \%$ por las mujeres, mientras que la respuesta no está dada por un 7,36\% por los hombres y un $1,81 \%$ por las mujeres.

En el siguiente gráfico se observarán las respuestas dadas por los encuestados a la pregunta número siete, lo cual en un porcentaje mayor los hombres piensan que las personas con discapacidad si son susceptibles a un rechazo laboral, mientras que en un porcentaje mayor las mujeres piensan que no son susceptibles a un rechazo. Este resultado se lo puede observar de forma gráfica en la figura 2.

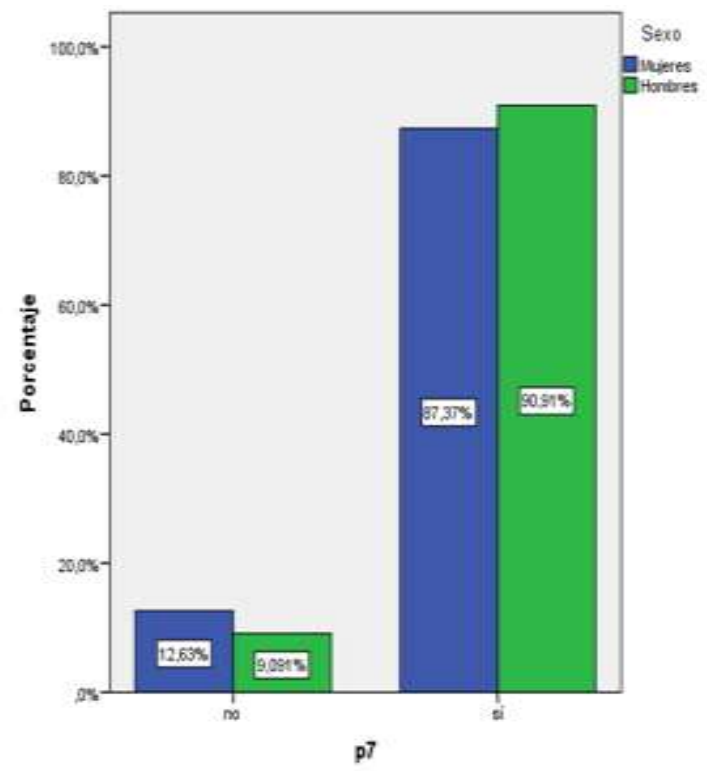

Figura 2.- Podemos observar que la respuesta si está representada en un $90,91 \%$ por los hombres, un

P. Guerra/C. Ramos/A. Subía/V. Carvajal/S. Borja
$87,37 \%$ por las mujeres, mientras que en la respuesta no está representada en un $9,01 \%$ por los hombres y un $12,63 \%$ por las mujeres.

En el siguiente gráfico podremos observar el porcentaje de discapacidades con más problemática en el ámbito laboral en base a las respuestas de los encuestados, lo cual en un porcentaje mayor, la población piensa que la discapacidad intelectual da más problemática y en un porcentaje menor la discapacidad sensorial, dejando en medio a la discapacidad física. Este resultado se lo puede observar de forma gráfica en la figura 3.

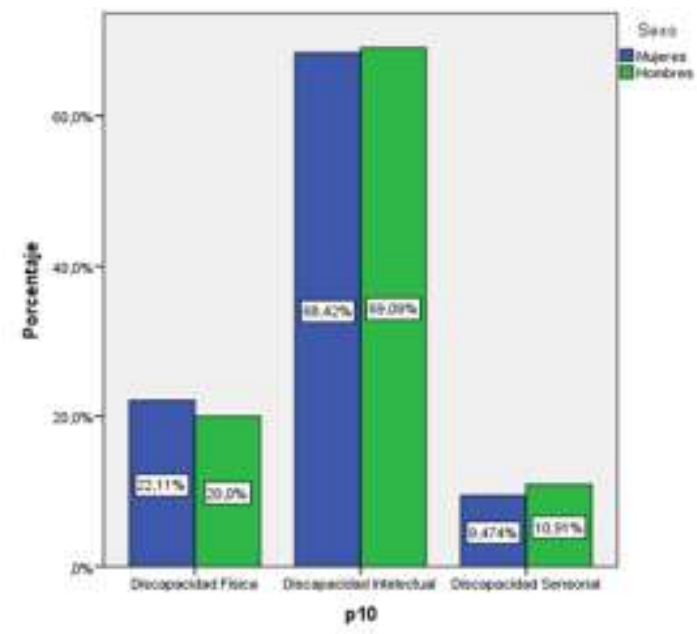

Figura 3.- Podemos observar que las personas de sexo masculino respondieron que la discapacidad intelectual da más problemática en el ámbito laboral con un $69,09 \%$ seguido de la discapacidad física con un $20 \%$ y la discapacidad sensorial con un $10,91 \%$, mientras que las mujeres determinaron que la discapacidad con más problemática es la discapacidad intelectual con un $68 \%$ seguido de la discapacidad física con un $22,11 \%$ y de la discapacidad sensorial con un $10,91 \%$.

En el siguiente cuadro observaremos el porcentaje de rendimiento laboral de las personas con discapacidad dicho por diferentes profesionales. Este resultado se lo puede observar de forma gráfica en la figura 4. 


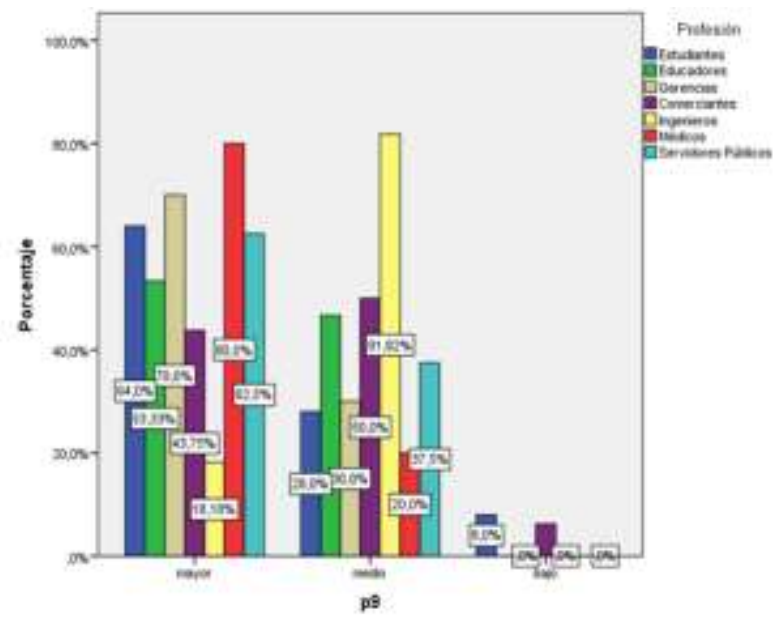

Figura 4.- En el cuadro podemos observar el desempeño laboral de las personas con discapacidad establecido por alto, medio y bajo. Alto un 64\% está representado por estudiantes, $53,33 \%$ por educadores, $70 \%$ por gerencia, $43,73 \%$ por comerciantes, $18,18 \%$ por ingenieros, $80 \%$ y el más alto por médicos y $62,5 \%$ por servidores públicos. Medio un $28 \%$ por estudiantes, $30 \%$ por educadores, $50 \%$ por comerciantes, $81,82 \%$ y el más alto por ingenieros, $20 \%$ por médicos y el $37,5 \%$ por servidores públicos. Bajo un $8 \%$ por estudiantes y un $6 \%$ por comerciantes.

En el siguiente cuadro observaremos el porcentaje por edades de personas que respondieron si las personas son o no susceptibles a un rechazo de parte de las organizaciones. Este resultado se lo puede observar de forma gráfica en la figura 5.

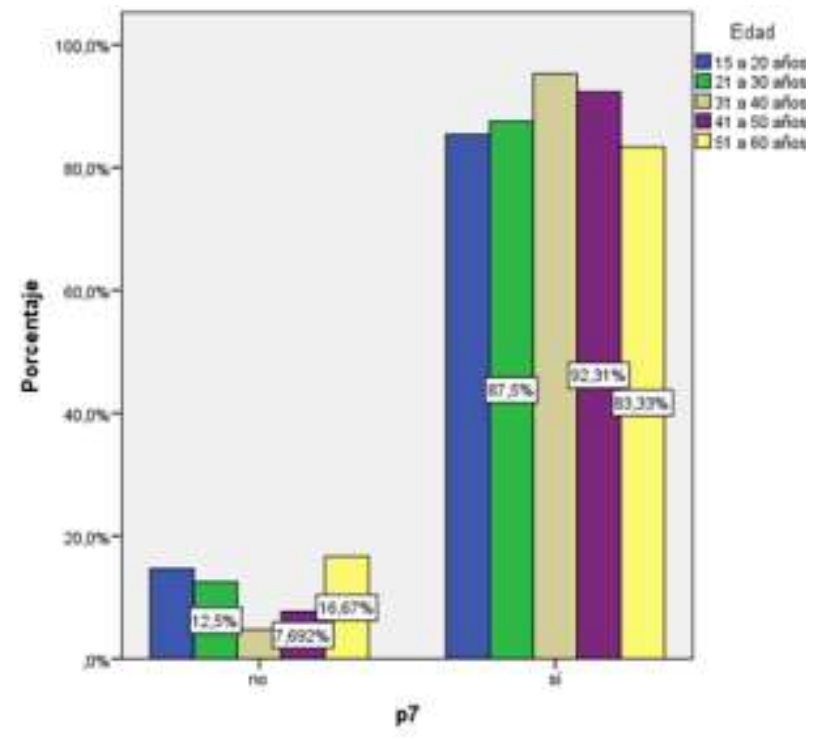

Figura 5.

\section{DISCUSIÓN Y CONCLUSIONES}

Los estudios realizados sobre inserción laboral de personas con necesidades especiales coinciden con otras investigaciones de diferentes países. Por ejemplo, en el Ecuador de acuerdo al artículo 330 de la constitución de la República indica que las personas con discapacidad tienen garantizada la inserción y accesibilidad en igualdad de condiciones al trabajo remunerado [7], lo cual tiene relación con los valores reflejados en el presente estudio donde la mayor parte de personas entrevistas afirman conocer acerca de los programas de inclusión laboral a personas con discapacidad.

Con respecto a la diferenciación en los procesos de selección en las personas con discapacidad dentro del presente estudio de investigación el mayor porcentaje de personas menciona que las personas con discapacidad deben tener un proceso de selección diferente, lo cual se relaciona con un estudio realizado por Valls et al [8] Las diferentes afirmaciones realizadas por los profesionales que se dedican a la inclusión laboral de personas con discapacidad nos hacen pensar que comienza a estar en crisis en el ámbito profesional la idea de que a través de la integración laboral se conseguirá automáticamente la integración social, las sociedad considera aceptable la inserción laboral pero existen aún presentes factores discriminatorios que crean diferencias desde la contratación de personal a grupos vulnerables.

Otro dato significativo dentro de esta investigación se encuentra dentro del rechazo que sufren las personas con necesidades especiales de lo cual un gran porcentaje concuerda en que efectivamente son rechazadas en el contexto laboral de la misma forma en el estudio realizado por Mercado et al [9] que mencionan que en su mayoría las personas con discapacidad sufren algún tipo de rechazo con una carrera de obstáculos debido a diversas circunstancias, tales como: el incumplimiento de la norma, el desconocimiento de las ayudas para la contratación del colectivo, la falta de credibilidad en sus capacidades, apoyo profesional y familiar.

En relación a dos preguntas realizadas en base al aporte de las personas con capacidades especiales a la organización así como también su rendimiento se concuerda que en ambos aspectos los porcentajes son positivos y por tanto altos es decir las personas discapacitadas aportan y trabajan efectiva y eficientemente para la empresa, este proceso no se podría facilitar según afirma el estudio realizado por Hernández et al [10], quienes indican que el éxito de la inclusión de los jóvenes discapacitados en un entorno laboral no depende de 
un factor único sino que se han de dar una serie de elementos que, de modo sinérgico, permitan una buena adaptación al puesto de trabajo y a un grupo social concreto. La formación laboral es una pieza clave, pero sin duda el plantear la incorporación al trabajo entendida como un proceso requiere coordinar diferentes elementos tales como: la

\section{REFERENCIAS BIBLIOGRAFICAS}

[1] Organización Mundial de la Salud (2016), [5 de Febrero del 2016]. Disponible en: http://www.who.int/topics/disabilities/es/

[2] Enable: Los derechos y la dignidad de las personas con discapacidad (2010), [5 de Febrero del 2016].Disponible en: http://www.un.org/spanish/disabilities/default.asp?i $\mathrm{d}=495$

[3] Manual de buenas prácticas para la inclusión laboral de personas con discapacidad (2013, [16 de febrero del 2016]. Disponible en: http://www.jardinuniversitario.utm.edu.ec/departa mento/media/areatecnica/buenas_practicas_inclusio n_laboral.pdf

[4] Organización Internacional del Trabajo: Discapacidad y Trabajo (2014), [16 de febrero del 2016]. Disponible

en: http://www.ilo.org/global/topics/disability-andwork/lang--es/index.htm

[5] Organización Internacional del Trabajo: Trabajo decente para personas con discapacidad: promoviendo derechos en la agenda global de desarrollo (2015), [18 de febrero del 2016]. Disponible en: http://www.ilo.org/wcmsp5/groups/public

[6] Organización Internacional del Trabajo: Estudio: factores para la inclusión laboral de las personas http://web.sofofa.cl

[7] Ordoñez, C. (2011) Breve análisis de inserción laboral de personas con discapacidad en el Ecuador. Revista de Educación 6 (2) 145-147.

[8] Valls , J. ; Villa, M.; Pallisera, M. (2003). La inserción de las personas con discapacidad en el trabajo ordinario. El papel de la familia.113-114 formación inicial laboral, el centro de trabajo (los compañeros y jefes han de entender cómo se han de relacionar), las familias y el propio trabajador.

[9] Mercado Garcia, E; García Vicente, L (2010). La inserción laboral de las personas con discapacidad: una salida social para trabajadores para trabajadores sociales.Red de Revistas Científicas de América Latina y el Caribe, España y Portugal 58

[10] Hernández Castilla, R.; Cerrillo, R.; Izuzquiza, D. (2008). La Inclusión de Discapacitados Intelectuales en el Mundo Laboral: Análisis Cualitativo.Estudio de un caso. Revista Latinoamericana de Educación Inclusiva 44-45 